\title{
Effect of External Contacts on Edge Magnetic Moments in Graphene Nanoribbons
}

\begin{abstract}
S. KROMPIEWSKI*
Institute of Molecular Physics, Polish Academy of Sciences, M. Smoluchowskiego 17, 60-179 Poznań, Poland

The main problem of interest of this study is the influence of external electrodes on the edge magnetic moments in graphene nanoribbons. The studies are carried out within the framework of tight-binding method (for $\pi$-state electrons) and the Landauer-Büttiker formalism combined with the Green function technique. It is shown that the edge atom moments get reduced (and eventually disappear) when the graphene nanoribbon/electrode interface becomes more and more transparent for electrons.
\end{abstract}

DOI: 10.12693/APhysPolA.127.523

PACS: 72.80.Vp, 73.63.Rt, 84.32.Dd

\section{Introduction}

A problem of carbon-based structures' magnetism is not free from controversies and still open to debate. Theoretically, it is well known that magnetic moments can be formed at carbon atoms with unsaturated bonds. In graphene nanostructures, typically this situation corresponds either to the zigzag-type fragments of the outer edges or the inner boundaries close to vacancies or holes [1-4]. In this contribution the former case is studied. The edge states of the graphene nanoribbon (GNR) are known to have very flat energy spectra (in the spin non-degenerate case) so that the so-called Stoner criterion is fulfilled and appearance of magnetic moments could be expected. In fact, recent experimental studies support this expectation. In particular, Ref. [5] shows that edge states reveal a pronounced spin splitting consistent with the Stoner theory.

\section{Methodology}

The setup under studies is depicted in Fig. 1. It consists of a GNR (black spheres) placed in the page plane $(x, y)$ and two 3-dimentional electrodes (only interface atoms are shown) perpendicular to it. Electronic, magnetic and electric transport properties of the setup can be easily described in terms of the tight-binding Hamiltonian (1) composed of the nearest neighbor hopping term, and the Stoner splitting term (2):

$$
H=-\sum_{i, j, \sigma} t_{i j}|i, \sigma\rangle\left\langle\sigma, j\left|+\frac{1}{2} \sum_{i, \sigma} \Delta_{i \sigma}\right| i, \sigma\right\rangle\langle i, \sigma|,
$$

where

$$
\Delta_{i \sigma}=U\left(n_{i \sigma}-n_{i-\sigma}\right) .
$$

Above, $U$ stands for the intraatomic Coulomb interaction parameter and $n_{i \sigma}$ is the number of carbon $\sigma$-spin

* corresponding author; e-mail: stefan@ifmpan.poznan.pl electrons at the $i$-th lattice site. The hopping integral $t_{i j}$ is equal to $-2.7 \mathrm{eV}$ and $t_{\mathrm{c}}$ (free parameter) for neighboring $\mathrm{C} / \mathrm{C}$ atoms, and neighboring carbon/electrode atoms, respectively.

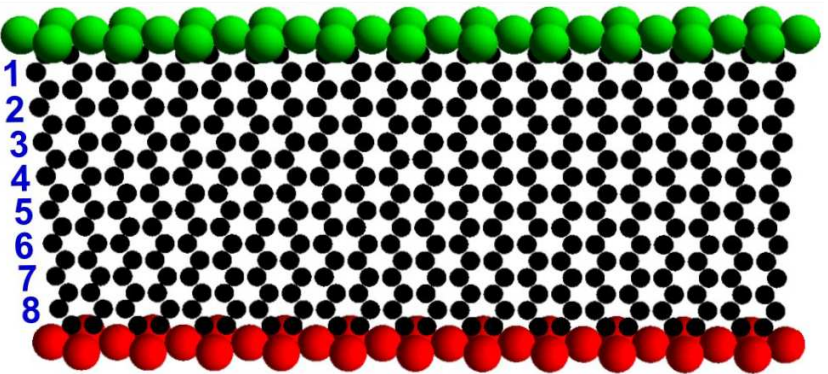

Fig. 1. Graphene nanoribbon (small spheres) sandwiched between external contacts (big spheres). The contacts are infinite in the $x-z$ plane and semiinfinite along the $y$ direction. Edge atoms on the lefthand side are enumerated.

In the absence of external contacts (or $t_{\mathrm{c}}=0$ ) one has to diagonalize Eq. (1) by solving the eigenequation

$$
(H-E)\left|u_{E}\right\rangle=0
$$

with the condition

$$
n_{i}=\sum_{E<E_{\mathrm{F}}}\left|u_{E}^{i}\right|^{2}
$$

If the electrodes are attached to the GNR than one has to solve self-consistently the following set of equations:

$$
\begin{aligned}
& G=\left(\hat{1} E-H-\Sigma_{1}-\Sigma_{2}\right)^{-1}, \\
& n_{i}=-\frac{1}{\pi} \int_{-\infty}^{E_{\mathrm{F}}} \mathrm{d} E \operatorname{Im} G_{i i}(E), \\
& \Sigma_{1}=T_{1} g_{1} T_{1}^{+}, \quad \Sigma_{2}=T_{2} g_{2} T_{2}^{+},
\end{aligned}
$$

where $G$ is the Green function matrix, $g_{1}$ and $g_{2}$ are surface Green functions of the source and drain electrodes, whereas $T_{1}, T_{2}$ are hopping matrices through interfaces (proportional to $t_{\mathrm{c}}$ ). For brevity the spin indexes in Eqs. (3) and (4) have been skipped. Details about surface Green function modelling can be found in Refs. $[6,7]$. 


\section{Results}

The main results of these studies are presented in Figs. 2 and 3. It is easily seen that magnetic moments can appear at the zigzag fragments of the edges. The calculations show that an antiparallel alignment of magnetic moments at the opposite edges is energetically more favorable than the parallel one.

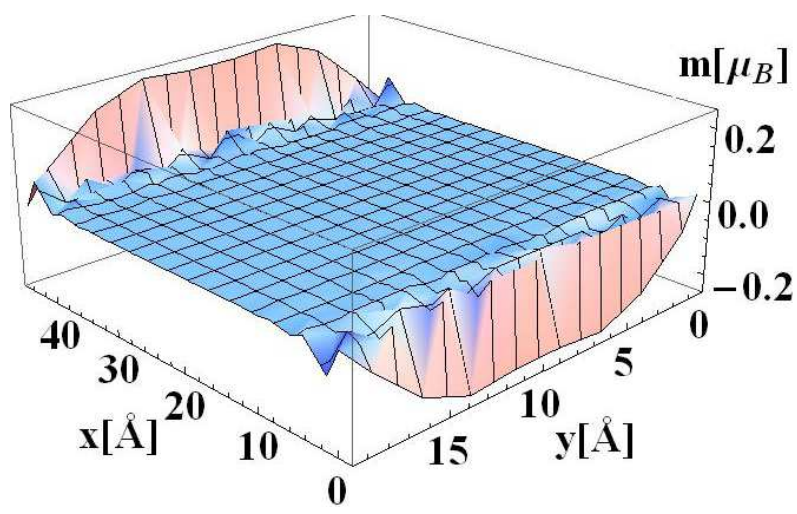

Fig. 2. Magnetization profiles of the GNR $19.7 \AA$ long and $45.4 \AA$ wide. The zigzag edges have pronounced magnetic moments antiparallel aligned to each other. The parameters are: $t_{\mathrm{c}} / t=0.25$ and $U / t=0.6$.

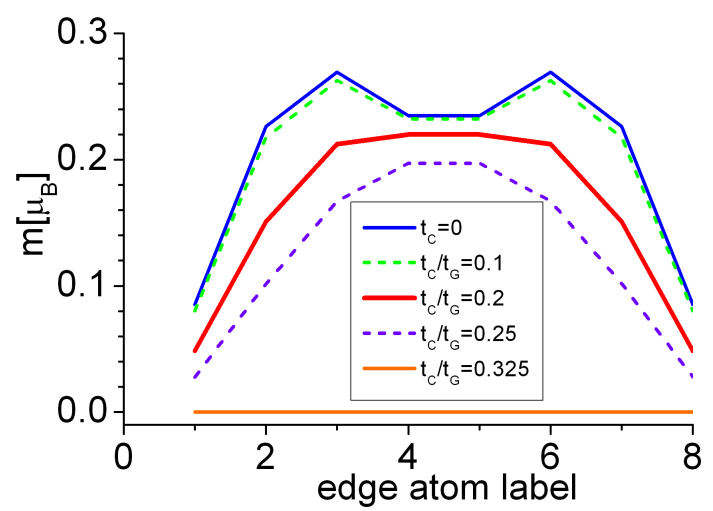

Fig. 3. Magnetic moments at successive edge atoms enumerated as in Fig. 1. The legends refer to the particular curves going from the top downwards.

Beyond the zigzag edges magnetic moments quickly vanish. Figure 3 shows that edge magnetic moments in the GNR can exist if the coupling with the external contacts is not too strong. For small and moderate values of the $t_{\mathrm{c}}$ parameter the edge magnetic moments can reach noticeable values (exceeding $0.2 \mu_{\mathrm{B}}$ for the present parameterization). Importantly, it results from the present studies that magnetic moments at the zigzag edges decrease and eventually completely disappear if the GNR/electrode interface transparency gets better and better. In particular, the edge magnetization decreases gradually up to $t_{\mathrm{c}} / t \approx 0.25$, and next collapses in an abrupt way. It was tested that the present findings concerning magnetic moments hold also qualitatively for different choices of parameters. It should be also stressed that the zigzag edges here are supposed to be hydrogenated, forming $s p^{2}$ type $\mathrm{C}-\mathrm{H}$ bondings. This is why the main attention can be directed just to itinerant $\pi$-electrons rather than to $\sigma$-states which lie far away from the charge neutrality point.

\section{Conclusions}

Summarizing, the present studies show that apart from the well-known reasons responsible for weakening or collapsing of edge magnetic moments due to e.g. temperature or edge reconstruction effects, also the contact effect plays an important role. The presence of the contacts results in broadening of electron energy levels and consequently to reduction or even disappearance of the edge magnetism in graphene nanoribbons. This finding makes it clear why edge magnetism in GNRs has not been detected so far for well electrically contacted setups.

\section{Acknowledgments}

This project was supported by the Polish National Science Centre from funds awarded through the decision No. DEC-2013/10/M/ST3/00488.

\section{References}

[1] I. Weymann, J. Barnaś, S. Krompiewski, Phys. Rev. B 85, 205306 (2012).

[2] X.W. Li, Q. Wang, Phys. Chem. Chem. Phys. 14, 2065 (2012).

[3] M. Wierzbicki, R. Świrkowicz, J. Barnaś, Phys. Rev. B 88, 235434 (2013).

[4] P.-H. Chang, B.K. Nikolic, Phys. Rev. B 86, 041406 (2012).

[5] C. Tao, L. Jiao, O.V. Yazyev, Y.-C. Chen, J. Feng, X. Zhang, R.B. Capaz, J.M. Tour, A. Zettl, S.G. Louie, H. Dai, M.F. Crommie, Nature Phys. 7, 616 (2011).

[6] S. Krompiewski, Phys. Rev. B 80, 075433 (2009).

[7] S. Krompiewski, Nanotechnology 23, 135203 (2012). 